\title{
The Importance of Analytical Chemistry in Therapeutic Drug Monitoring for Personalized Medicine
}

\author{
Hamza Sofiyev $^{1 *} \quad$ Neşet Neşetoğlu ${ }^{1,2 \underline{D}}$ Ibrahim Danışs Cem Kaplan $^{2} \quad$ Merve Keşkek Arslan ${ }^{2}$ \\ Serap Sağlık Aslan ${ }^{1 \underline{D}}$ Durişehvar Özer Ünal ${ }^{1,2 \underline{i} \underline{D}}$ \\ 1.Istanbul University, Faculty of Pharmacy, Department of Analytical Chemistry, 34116, Beyazit, Istanbul, \\ Turkey \\ 2.Istanbul University, Drug Research and Application Center, 34116, Vezneciler, Istanbul, Turkey
}

\begin{abstract}
Personalized therapy (PM) has the potential to adapt treatment with the best response and highest safety to provide better patient care. Key data is drug concentration of biological materials such as plasma and serum.Individual drug therapy means, choice of a drug and its dose regime should fit every individual specifically. Thus efficacy of a drug treatment would improve significantly. When developing an analytical method for (Therapeutic drug monitoring) TDM, it is important to choose a clinically relevant calibration range. This quantitation range should be built around the proposed target concentration, covering majority of samples as seen in the clinic (CiocanCartita et al. 2019).Inter-individual variability in Pharmacokinetic variables may affect the blood concentration of drug so TDM approaches could solve the dosing problem.To achieve individual drug therapy with a reasonably predictive outcome, one must further account for different patterns of drug response among geographically and ethnically distinct populations.
\end{abstract}

Keywords: LC-MS/MS, Therapeutic Drug Monitoring, Lenalidomide, Anastrozole

DOI: $10.7176 / \mathrm{CMR} / 12-7-05$

Publication date:September $30^{\text {th }} 2020$

\section{Introduction}

1.1 Therapeutic Drug Monitoring

Newer concepts of precision medicine such as Pharmacogenetics is the sub-branch of pharmacology, which examines the relationship between the individual's genetic tendencies and the body's individual response to drugs. Pharmacogenetic variability is major confounding factor in traditional weight base dosing or fixed dose. Personalized medicine and drug monitoring one potentially increasing area of drug therapy in recent years (CiocanCartita et al. 2019). Therapeutic Drug Monitoring (TDM) is done by collection of blood samples. Specific relative time to administration of final dose in practice and the goal of TDM to individualize blood drug concentrations to manage a patient's dose regimen and optimize patient benefit. The drug or drug metabolite concentrations can be measured and identified in the biological sample and compared to a target range or anticipated pharmacokinetics for the drug. To achieve an effective result, the criteria for TDM must be met: (1) Drugs with narrow therapeutic index (difference between minimum effective dosage and toxic dosage), (2) good correlation and effect between blood concentration, (3) high risk of side effects (4) there should be serious consequences for low or overdose, (5) extensive changes in metabolism, and (6) the effect of TDM test results on clinical outcomes should be interpretable and feasible.

Most of the drugs that meet most of the TDM criteria but are not particularly useful in measuring drug concentrations. For example, when a non-biologically active form of the drug is administered as a pro-drug, it can be metabolized in the body to produce the biologically active form of the drug. The prodrug administered is inert and the metabolite of the prodrug is an active substance. Most drugs display linear kinetics, but some drugs that exhibit nonlinear kinetics and require small incremental dose adjustments. Therefore, the determination of the drug administered does not guide treatment, therefore the active metabolite of the prodrug (eg, Irinotecan, tamoxifen, voriconazole and tacrolimus) should be determined for meaningful drug monitoring (Clarke \& Dasgupta 2016).

While developing an analytical method for TDM, it is important to choose a clinically appropriate calibration interval. This quantitation range should be evolved around the recommended target concentration, which covers majority of samples as observed in the clinic (Momper \& Wagner 2014).

Inter-individual variability in Pharmacokinetic variables including drug absorption, distribution metabolism and excretion may affect the blood concentration of drug so Therapeutic Drug Monitoring approaches could solve the dosing problem.

Concentration of drugs is minimum $\left(\mathrm{C}_{\mathrm{min}}\right)$ just before the dose is taken, then rises to a maximal concentration $\left(\mathrm{C}_{\max }\right)$ at a certain time after the dose (Tmax). The concentration then the decline becomes shallower Cmin as the amount of drug remaining falls. The area under the concentration-time curve (AUC) describes total drug exposure during the administred dosing interval (Fig. 1). 


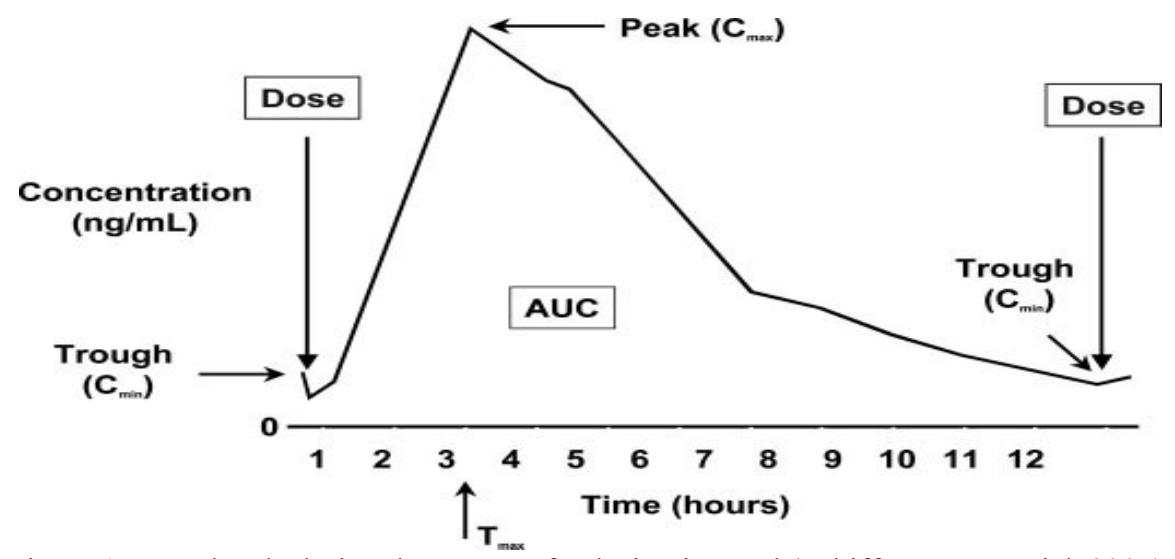

Figure 1. Drug levels during the course of a dosing interval (Schiff \& Cantarovich 2007).

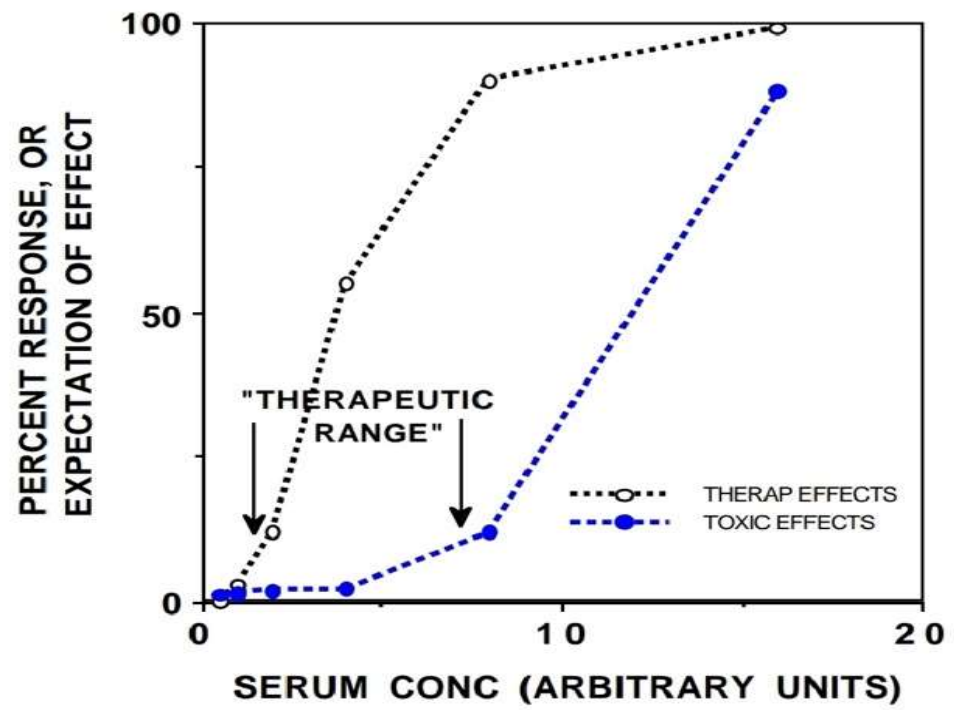

Figure 2. Therapeutic ranges (Hempel 2004).

Figure 2 shows the usual means of imaging of therapeutic ranges. First, it is first stated that the therapeutic effects have an "important" incidence with increased blood drug concentrations. This works as the beginning of the therapeutic range. It becomes "meaningful" and is considered "toxic range" (Hempel 2004).

The beginning of a therapeutic range is usually set at the first bend (left arrow) at the line where the rate of increase in the incidence of therapeutic events becomes noticeably greater than before. Similarly, the upper limit (right arrow) is usually taken in bending the line, which represents the relationship between serum concentration and the rate of increase in toxicity incidence (Ranganathan et al. 2019).

Each patient has a unique set of changes in the genome such as transcriptome, proteom and metabolom. Due to these changes, the individual may respond differently to treatment and lead to implicit adaptation steps in the therapeutic approach (Ciocan-Cartita et al. 2019).

Certain diseases such as Epilepsy, Organ Transplantation, Cardiology (Antiarrhythmic Drugs), Psychiatry, Infectious Disease, Oncology have spesific clinical conditions, and patient-specific clinical factors such as age, weight, renal function, drug interactions, plasma drug concentrations, and diet require routinely TDM. Most of the drugs used in the treatment of chronic diseases such as cardiac drugs, Antiepileptics, Antibiotics, Immunosuppressants, Anticancer drugs, Psychiatric drugs, but some toxic drugs such as aminoglycosides and vancomycin require more than 2 days.

TDM has many opportunities for the spread and development of the concept of personalized or sensitive treatment. Increased focus on specific populations such as age-related (eg pediatric or geriatric medicine) or disease-related (eg transplantation or cystic fibrosis) or health-defined (eg obesity) is also important for the effectiveness of treatment. as drug concentrations (Clarke \& Dasgupta 2016). 
Every person has own pathological changes inside. For ontological therapeutics, studies should be done for personalized medicine in the future. This way we can find better medication strategy for cancer type (CiocanCartita et al. 2019).

Determination of drug concentration in a particular human biological matrix is one of the most critical components of therapeutic drug monitoring. Many analytical procedures have been developed for TDM. As analytical techniques and instrumentation have advanced, this area of TDM has probably grown more rapidly than any other. These "sophisticated" techniques include various types of immunoassay, gas chromatography (GC), high performance liquid chromatography (HPLC), electrophoresis and liquid chromatography-mass spectrometry (LC-MS) (Xu \& Madden 2011).

The increased role of liquid chromatography-tandem mass spectrometry in therapeutic drug monitoring is based on advances in analytical instrumentation, thus providing a unique specificity, rapid and accurate, extreme sensitivity, high efficiency, the ability to simultaneously analyze multiple drugs and metabolites in one drop. The use of multiplex platforms for rapid detection of blood and single analytes in a few minutes. However, analysis with LC-MS / MS does not automatically provide reliable results and superiority over other tests (Shipkova \& Svinarov 2016).

Analysis of drugs in biological materials is very important and can provide valuable information about pharmacokinetics, pharmacodynamics, pharmacology, toxicology and TDM purposes. So far, in biological fluids, namely blood, urine, milk, etc. A wide variety of analytical methods have been developed and validated for the identification of drugs.

LC-MS / MS, tandem LC with MS / MS detection system has been found to be the best technique for traces of traces in biological fluids (Hempel 2004).

The LC-MS systems has become the most important analytical means nowadays for drug analysis in biological matrices. Contrary to GC-MS, LC-MS has small analyte limitations that can analyze polar, thermally labile and high molecular weight compounds without the derivatization step. Reductive and oxidative biotransformation of target compounds in phase I metabolism and conjugation products with glucuronic acid in phase II metabolism such as phase II metabolites, antibiotics, amino acids and peptides can be easily measured with high precision (Hempel 2019).

The sample preparation stage in LC-MS can be simplified compared to GC-MS, but this stage of analysis cannot be completely eliminated, because many substances contained in the sample extract can contaminate the system and have a matrix effect that can lead to inaccurate or false negative results mainly in qualitative analysis. In some situations, a derivatization step can be used. Commercially available instruments allow sample preparation procedures to be done online with the LC-MS system, providing high throughput samples (Hempel 2004).

MS detection provides greater specificity and selectivity than most other detectors used in chromatography, but the MS detector is more expensive than others and is often not available in laboratories. There are also issues specific to LC-MS, including discussions on identifying the appropriate number of ions and "acceptable" ion ratios to be evaluated for working with MS and the selection of qualitative and quantitative ions.

Despite these concerns, LC-MS and LC-MSMS can provide significant advantages over methods with GCMS. Sample preparation steps can often be simplified or eliminated with LC, which reduces both labor and solvent use. In addition, while developing methods for determining polar metabolites without prior derivatization for analysis in GC, pre column extraction is possible in some LC systems. Dams et al. It also showed that it is appropriate to prepare urine samples by simple dilution in LC-MSMS. These advantages explain the increasing tendency for the development of opioid and other drug analysis from urine, especially with the detection of LC, MSMS (Clarke \& Dasgupta 2016).

Quadrupole mass analyzers are besides used in LC-MS, chiefly in their triple quadrupole hybrid configuration and has this combined system provides more accurate mass and excellent dynamic range and mass resolution. But the use of single quadrupole equipment, which is capable of operating as a mass filter for the selection of specific ions based on their mass-to-charge ratio is limited in drug analysis on account of low selectivity and sensitivity. As previously mentioned, the QQQmass spectrometers are excellent devices for quantitative analysis and identification of drugs and metabolites in biological samples. The combination of LCMS/MS system with ESI (or APCI) and QQQ mass analyzer provides better results than GC-MS/MS, since the LC-MS/MS ionization process is soft, identification, including ion annotation, spectral interpretation and matching with little or no fragmentation at the ionization source (Xu \& Madden 2011).

In this way, The first mass filter, Q1, separates ions by $\mathrm{m} / \mathrm{z}$ ratios or filters and passes only one ion into the collision cell, and the shredded ion enters Q2 and collides with neutral gas molecules. Fragmented ions are produced and passed to Q3 and a spectrum is obtained (Yao et al. 2013).

Therapeutic drug monitoring is an effective way of blood concentration of drugs, so lenalidomide which is used for treatment of myeloma and anastrozole is used for breast cancer. HPLC-MS/MS methods developed for the determination of those two drugs from plasma and serum. 


\subsection{Personalized Medicine}

It is difficult to argue that clinical medicine contributes greatly to our ability to treat and treat patients. However, whether medical care transforms morbidity and mortality patterns at the population level and to what extent it contributes to health and survival has been the subject of debate for over a century.This debate has taken on renewed importance as the scientific leadership at the all National Institutes of Health (NIH) on the World, Research Institutes and universities have taken up the challenge of personalized or precision medicine (Obasogie \& Darnovsky 2018).

When describing personalized medicine, this does not imply the creation of patient-specific drugs or medical devices, but rather the ability to classify individuals into subpopulations that differ in their predisposition to a particular disease.

This demonstrates the clinical practicality of using personalized medicine as sensitive medicine: the technology of testing the drug's biomarkers is becoming increasingly suitable for both efficacy and toxicity, while a layered approach to treatment for fewer patients (Donev 2016).

Precision medicine is supported by patient data. Health records and genetic codes of patients and healthy volunteers are vital and help people influence their own health care and research aspects (Hodson 2016).

\section{Materials And Methods}

\subsection{Lenalidomide}

Lenalidomide, an immunomodulatory drug, is a synthetic compound obtained by modifying the chemical structure of thalidomide to increase its potency and reduce its side effects. LENA is used as a treatment for myeloma and blood disorders called myelodysplastic syndromes. It is inhibits TNF-alpha production, stimulates $\mathrm{T}$ cells and indicated for the cure of transfusion-dependent anemia. It is also used in combination with dexamethasone for the treatment of multiple myeloma patients when other drug options are insufficient or inadequate. LENA has a short half-life (3-4 h) and does not accumulate in the plasma after repeated doses. The Cmax was $390 \mathrm{ng} / \mathrm{ml}$ for young and $568 \mathrm{ng} / \mathrm{ml}$ for older adults in the clinical trial. Its pharmacokinetics are consistent across patient populations, regardless of the type of hematologic malignancy. For Lenalidomide, a highly sensitive and high performance liquid chromatography tandem mass spectrometry (LC-MS/MS) assay is developed and also validated to the quantitate in human plasma. Liquid-liquid extraction method is used to extract Lenalidomide from human plasma. For analytical column and flow, reversed phase isocratic elution is used to anlyze. Mobile phase consists of methanol: $0.1 \%$ formic acid: $(90: 10 \%, \mathrm{v} / \mathrm{v})$. Detection was performed on LC-MS/MS which has triple quadrupole and use electrospray ionization in negative mode.

The calibration curve regression of lenalidomide was a linear regression model. Pomalidomide was used as the Internal Standard (IS). As a result, the optimal and stability coefficient $\left(\mathrm{r}^{2}\right)$ for validation was obtained and was in the acceptable range greater than 0.99. The average value for $r^{2}$ was found to be 0.99976 . Calibration curve's equation is $\mathrm{y}=0.001 \mathrm{x}-0.0173$. Concentration range $(20-1000 \mathrm{ng} / \mathrm{ml})$ for LENA was found to be accurate and precise respectively (Unpublished data -1).

\subsection{Anastrozole}

Anastrozole is the active substance used in breast cancer as a non-steroidal aromatase inhibitor. It inhibits estrogen synthesis in estrogen-dependent breast cancer cases. It is one of the leading drugs in cancer treatment by the World Health Organization (WHO). Therefore, the determination of anastrozole from human plasma is important for examining the therapeutic effect. For this purpose, the analysis of anastrozole from plasma was performed by liquid chromatography Tandem Mass Spectrometry. Separation was carried out on the Poroshell column using Solution B: ACN ( 85:15 solution A) mobile phase with Solution A: 5 mM NH 4 Ac and 0.1\% HAc, pH: 4.0 (50:50, $\mathrm{v} / \mathrm{v})$. Anastrozole was withdrawn from the plasma by liquid-liquid extraction using Tolterodine as the internal standard. For anastrozole and tolterodine, m / z $294 \rightarrow 225$ and $284 \rightarrow 197$ values were selected, respectively. R: 0.9951 was found in the linear range of $0.550 \mathrm{ng} / \mathrm{ml}$. The method was validated and plasma levels were determined in breast cancer patients which using anastrozole.

The $C_{\max }$ was $15 \pm 2.3 \mathrm{ng} / \mathrm{ml}$ in the clinical trial. The calibration curve regression of anastrozole was a linear regression. This result gave the best determination coefficient $\left(\mathrm{r}^{2}\right)$ for validation and was greater than 0.99 , which was in the acceptable range. The average value for $\mathrm{R}^{2}$ was found to be 0.9999 . Calibration curve's equation is $\mathrm{y}=1.5 \times 10-5 \times 0.022$. The concentration range of anastrozole $(0.5$ to $50 \mathrm{ng} / \mathrm{mL})$ was found to be accurate and precise.

These results indicate that the method has good precision and accuracy are within the acceptance limit of $<$ $15 \%$ and $\pm<15 \%$ for precision and accuracy respectively. Recovery was $87.68 \%$ (Neşetoğlu et al. 2020).

\section{Discussion}

LC - MS / MS is a very useful tool for therapeutic drug monitoring, particularly if there are problems such as metabolite interactions or lack of a suitable chromophore (UV absorbing functional group) group to absorb light 
for UV detection to provide an alternative method. LC - MSMS analysis is possible with the simple sample preparation stage, as well as more automated methods such as Solid Phase Extraction (SPE) systems and pipetting robots that can perform liquid-liquid extraction very simply and rapidly. Multiple analyzes that enable simultaneous analysis of ordinarily prescription concomitant medications drugs, such as antiretrovirals or immunosuppressants, can be concluded faster than individual analyzes when the need for multiple drug preparation steps are minimized.

The use of matrices other than blood or serum is becoming more and more popular for TDM. Saliva analysis makes it possible to measure free drug levels that are important for the drug, which is highly bound to plasma proteins such as phenytoin, since only the free part of the drug is physiologically active.

The determination of the unbound drug fraction in the blood is very difficult and time-consuming due to the easily discomposed poise between free and bound drug during sample preparation. Saliva analysis may be an easier way to measure the extent of active drugs in the body, since protein-dependent drugs cannot enter saliva. Hair analysis is proposed as a way of monitoring the patient's suitability for a long-term patient's prescription medication. Hair analysis has not yet been identified as a suitable matrix for TDM, but it may be a convenient way to monitor patients in the future when the relationship between the hair and plasma levels of drugs is established and simple methods of sample preparation develop. The use of dried blood stains for TDM is also becoming more popular because analysis of dried blood stain (DBS) allows patients to take samples at home and send them to the laboratory. This can be much more available for the patient and even enable AUC analysis for precise drug monitoring without having to spend the day in the hospital.

Development of an analytical method is required for TDM. A clinically appropriate calibration interval is required. These quantity ranges are important, including some samples, as in the clinic (5). Lenalidomide maximum blood concentration is $568 \mathrm{ng} / \mathrm{mL}$ found in adults the developed and validated methods calibration standards range were established $20-1000 \mathrm{ng} / \mathrm{mL}$. This Calibration curve range was covering the sample seen in clinic. It is used in the treatment of early-stage breast cancer in postmenopausal women with a positive hormone receptor and treatment of advanced breast cancer in postmenopausal women. For anastrozole maximum blood concentration is $15 \pm 2.3 \mathrm{ng} / \mathrm{mL}$ found in adults the developed and validated methods calibration standards range were established $0.5-50 \mathrm{ng} / \mathrm{mL}$.

\section{Conclusion}

As a result, LC-MSMS has made it possible for TDM to be analyzed in multiple matrices, such as blood, hair and saliva level of many drugs that cannot be measured. LC - MSMS sample preparation method and careful selection of the internal standard, and complete verification of the analyzes will prevent most of the problems and make it possible to determine the correct amount of medication (Unpublished-1).

More recently, LC-MSMS has replaced other chromatographic methods by analyzing multiple compounds at once from a single sample for screening and validation of genetic disorders and congenital metabolism diseases.

It is particularly advantageous given the limited sample volume typically obtained from newborns, thanks to the use of several dozen species of hereditary amino acids and a small sample for metabolic disorders.

\section{References}

Ciocan-Cartita, C.A., Jurj, A., Buse, M., Gulei, D., Braicu, C., Raduly, L., et al. (2019), "The Relevance of Mass Spectrometry Analysis for Personalized Medicine through Its Successful Application in Cancer "Omics" ", Int J Mol Sci.20(10), MDPI, 2576.

Clarke, W., Dasgupta, A., (2016), "Special populations, physiological conditions and pharmacogenomics", Clinical challenges in therapeutic drug monitoring, Elsevier.

Donev, R., (2016), Advances in Protein Chemistry and Structural Biology, 102, Elsevier, 81, 188, 189.

Hempel, G., (2004), "Drug monitoring and clinical chemistry", Elsevier.

Hempel, G., (2019), Methods of Therapeutic Drug Monitoring Including Pharmacogenetics, Elsevier.

Hodson, R., (2016), "Precision medicine", Nature, 537(7619), 49.

Momper, J.D., Wagner, J.A. (2014), “Therapeutic Drug Monitoring as a Component of Personalized Medicine: Applications in Pediatric Drug Development". Clinical Pharmacology \& Therapeutics, 95(2), ASCPT, 138140.

Neşetoğlu, N., Kaplan, C., Aslan, S.S., and Ünal, D.Ö., (2020), Pak. J. Anal. Environ. Chem, 21(1).

Obasogie, O.K., Darnovsky, M. (2018), “Beyond Bioethics”, Toward a New Biopolitics, University of California Press.

Ranganathan, P., Gunasekaran, V., Singhvi, I., Ansari, M.J. (2019), Saudi J Biol Sci, 26(7), 1843.

Schiff, J., Cole, E., Cantarovich. M. (2007), "Therapeutic Monitoring of Calcineurin Inhibitors for the Nephrologist". Clin J Am Soc Nephrol. 2(2), CJASN, 374.

Shipkova, M., Svinarov, D., (2016), “LC-MS/MS as a tool for TDM services: Where are we?”. Clin Biochem, 49(13), 1009-1023. 
Xu, Q.A., Madden, T.L.. (2011), Analytical methods for therapeutic drug monitoring and toxicology, Wiley.

Yao, X., McShane, A.J., Castillo, M.J. (2013), "Chapter 17 - Quantitative Proteomics in Development of Disease Protein Biomarkers". In: Issaq HJ, Veenstra TD, editors. Proteomic and Metabolomic Approaches to Biomarker Discovery. Boston: Academic Press, 259-78. 